\title{
Predation with Indirect Effects in Fluctuating Environments
}

\author{
Tomás Caraballo • Renato Colucci . \\ Xiaoying Han
}

Received: date / Accepted: date

\begin{abstract}
We investigate the long term dynamics for a predation model of Plankton community with indirect effects, under fluctuating environments. A random version and a stochastic version with multiplicative noise of the model are discussed and compared. We prove that the solutions to both versions are non-negative and bounded given any non-negative positive initial conditions. We also prove that both the random system and the stochastic system possess a unique random attractor under the same set of assumptions, by using the classical theory of random dynamical systems. In addition we provide conditions under which coexistence of species exists for the random system.
\end{abstract}

Keywords random dynamical system · population dynamics · random attractor $\cdot$ indirect effects.

Mathematics Subject Classification (2000) Primary 92D25 · Secondary $34 \mathrm{C} 60$

This work has been partially supported by FEDER and the Spanish Ministerio de Economía y Competitividad project MTM2011-22411 and the Consejería de Innovación, Ciencia y Empresa (Junta de Andalucía) under grant 2010/FQM314 and Proyecto de Excelencia P12FQM-1492.

T. Caraballo

Departamento de Ecuaciones Diferenciales y Análisis Numérico, Universidad de Sevilla Apdo. de Correos 1160, 41080 Sevilla, Spain

E-mail: caraball@us.es

R. Colucci

Department of Mathematical Sciences,

Xi'an Jiaotong-Liverpool University

111 Ren'ai Road, SIP, Suzhou P. R. China 215123

E-mail: renatocolucci@hotmail.com

X. Han

Department of Mathematics and Statistics,

221 Parker Hall, Auburn University

Auburn, AL 36849, USA

E-mail: xzh0003@auburn.edu 


\section{Introduction}

Indirect effect refers to species interactions which can occur through chains of direct species interaction, such as predation or interference competition. The studies of indirect effects are of great importance to the biology and ecology communities, as they can link the population dynamics of species that do not interact directly (see $[3,4,6,12,15,17,18]$ and references therein). The following mathematical model has been introduced to study indirect effects of predation in a Plankton community (see [10] and [11]):

$$
\left\{\begin{array}{l}
\dot{Z}=Z\left(-e+u_{c} C+u_{g} G\right), \\
\dot{C}=C\left[a_{c} I_{0}-\left(a_{c}+u_{c}\right) Z-a_{c} C-a_{c} G\right]-\mathcal{I}_{1}(Z, C, G), \\
\dot{G}=G\left[a_{g} I_{0}-\left(a_{g}+u_{g}\right) Z-a_{g} C-a_{g} G\right]+\mathcal{I}_{2}(Z, C, G) .
\end{array}\right.
$$

where $Z$ represents the density of a population of Zooplanckton (Predator), $C$ and $G$ represents the densities of two populations of Phytoplanckton (Preys) with different sizes, $e, u_{c}, u_{g}, a_{c}$ and $a_{g}$ are nonnegative parameters, and $\mathcal{I}_{1}$ and $\mathcal{I}_{2}$ are functions describing the indirect effects. A typical choice of $\mathcal{I}_{1}$ and $\mathcal{I}_{2}$ is

$$
\mathcal{I}_{i}(Z, C, G)=m_{i} C Z, \quad m_{i}>0, \quad i=1,2,
$$

which indicates that the predator prefers to predate the prey of smaller size $(C)$ and the other group of prey $(G)$ takes advantage of it.

System (1) is an autonomous system with constant parameters, for which traditional stability analysis relies on the concept of equilibriums or steady states. For example, it has been proved in [10] by stability analysis that there is a large region of variation of parameters for which any positive solution of (1) exhibits coexistence. However, most natural populations fluctuate greatly with respect to the variation of their environments, and may exhibit dynamical patterns within observable times that cannot be fully characterized by stationary steady states. Various replacements for the equilibrium exist, such as limit cycles, strange attractors and stationary probability distributions. For example, the attractor of the system has been studied in [11] by bifurcation theory, and turned out to be an interior fixed point or a limit cycle. Nevertheless, these alternatives are merely equilibria on different time scales, and do no apply to systems with time-varying or random parameters due to fluctuating environments.

Mathematically, the time-varying or random parameters preclude convergence of a system to a traditional steady state. Instead, the population process may converge on a time-dependent or random object, which is independent of the initial population densities. In the context of dynamical systems, this convergence may occur in two senses, the forward or the pullback sense. In the forward sense, the difference between the population process and limiting object approaches zero as time $t$ increases. In the pullback sense, the dependence of population density on a starting density is lost as the starting time recedes 
into the far past. The pullback attractor is a concept well suited to population models including system (1), when one observes at a certain time the system which has been running "from ages". Therefore the newly developed mathematical theory of non-autonomous and random dynamical systems $[1$, 14] becomes powerful tools to analyze the asymptotic dynamics of population models, in temporarily-varying or random environments.

In [7], we studied system (1) in time-varying environments by introducing seasonal, i.e., time-dependent indirect effect functions of the form

$$
\mathcal{I}_{i}(Z, C, G)=g_{i}(t) C Z, \quad 0 \leq g_{i}(t) \leq G_{i}, \quad G_{i} \in \mathbb{R} \quad i=1,2,
$$

and investigated the existence of uniqueness of a pullback attractor, upper bound of its dimension, as well as its coexistence structure, by using the theory of non-autonomous dynamical systems. In this paper we will study the long term dynamics of system (1) under random environments, by using the theory of random dynamical system. The randomness of environments will be characterized in two different means: introducing random parameters or introducing stochastic noise terms in the system.

Let us write system (1) with indirect effect (2) in a more general format:

$$
\left\{\begin{array}{l}
\dot{x}(t)=x(t)\left(-b_{1}+a_{12} y(t)+a_{13} z(t)\right), \\
\dot{y}(t)=y(t)\left(b_{2}-a_{21} x(t)-a_{22} y(t)-a_{23} z(t)\right)-m_{1} x(t) y(t), \\
\dot{z}(t)=z(t)\left(b_{3}-a_{31} x(t)-a_{32} y(t)-a_{33} z(t)\right)+m_{2} x(t) y(t),
\end{array}\right.
$$

where all the parameters $a_{i, j}(i, j=1,2,3), b_{j}(j=1,2,3), m_{j}(j=1,2)$ are nonnegative. We will first study its random counterpart by considering that the carrying capacities $b_{j}$ are affected or perturbed by random environmental influences modeled by the paths of a probability space $(\Omega, \mathcal{F}, \mathbb{P})$, in other words, we will study the system:

$$
\begin{aligned}
& \dot{x}(t, \omega)=x(t)\left(-B_{1}\left(\theta_{t} \omega\right)+a_{12} y(t)+a_{13} z(t)\right), \\
& \dot{y}(t, \omega)=y(t)\left(B_{2}\left(\theta_{t} \omega\right)-a_{21} x(t)-a_{22} y(t)-a_{23} z(t)\right)-m_{1} x(t) y(t), \\
& \dot{z}(t, \omega)=z(t)\left(B_{3}\left(\theta_{t} \omega\right)-a_{31} x(t)-a_{32} y(t)-a_{33} z(t)\right)+m_{2} x(t) y(t),
\end{aligned}
$$

where the properties of the random parameters $B_{j}\left(\theta_{t} \omega\right),(j=1,2,3)$ will be specified later. Essentially, these equations are random ordinary differential equations which can be solved for each $\omega \in \Omega$ fixed. In other words, we have a system of ordinary non-autonomous differential equations depending also on a random parameter (see, e.g. [5], [14]). Secondly, we will also consider its stochastic counterpart

$$
\begin{aligned}
\mathrm{d} X(t)= & X(t)\left(-b_{1}+a_{12} Y(t)+a_{13} Z(t)\right) \mathrm{d} t+\mu X(t) \circ \mathrm{d} W(t), \\
\mathrm{d} Y(t)= & Y(t)\left(b_{2}-a_{21} X(t)-a_{22} Y(t)-a_{23} Z(t)-m_{1} X(t) Y(t)\right) \mathrm{d} t \\
& +\mu Y(t) \circ \mathrm{d} W(t), \\
\mathrm{d} Z(t)= & Z(t)\left(b_{3}-a_{31} X(t)-a_{32} Y(t)-a_{33} Z(t)+m_{2} X(t) Y(t)\right) \mathrm{d} t \\
& +\mu Z(t) \circ \mathrm{d} W(t),
\end{aligned}
$$


where $\mu$ is a non-negative constant, $W(t)$ is a standard Wiener process on a probability space and $\circ$ denotes the Stratonovich sense of stochastic integration. We note that the equivalent Ito stochastic differential equations are obtained by adding the quantity $\frac{1}{2} \mu^{2}$ to the $b_{j}$ coefficients. Namely the system (6)-(8) becomes

$$
\begin{aligned}
\mathrm{d} X(t)= & X(t)\left(-b_{1}+\frac{1}{2} \mu^{2}+a_{12} Y(t)+a_{13} Z(t)\right) \mathrm{d} t+\mu X(t) \mathrm{d} W(t) \\
\mathrm{d} Y(t)= & Y(t)\left(b_{2}+\frac{1}{2} \mu^{2}-a_{21} X(t)-a_{22} Y(t)-a_{23} Z(t)-m_{1} X(t) Y(t)\right) \mathrm{d} t \\
& +\mu Y(t) \mathrm{d} W(t) \\
\mathrm{d} Z(t)= & Z(t)\left(b_{3}+\frac{1}{2} \mu^{2}-a_{31} X(t)-a_{32} Y(t)-a_{33} Z(t)+m_{2} X(t) Y(t)\right) \mathrm{d} t \\
& +\mu Z(t) \mathrm{d} W(t)
\end{aligned}
$$

and the main difference with the random system (3)-(5) is that the latter cannot be solved pathwise.

In particular, we will prove the existence of a random pullback attractor for systems (3) - (5) and (6) - (8), along with some detailed properties of the attractor.

The rest of this paper is organized as follows. In Section 2 we will recall a brief preliminary review of classical random dynamical system (RDS) theory, in Section 3 we will analyze the random system (3) - (5). In Section 4 we will analyze the stochastic system $(6)-(8)$. Some closing remarks will be stated in Section 5.

\section{Preliminaries}

In this section we first present some concepts (from [1]) related to general random dynamical systems (RDSs) and random attractors that we require in the sequel.

Let $\left(X,\|\cdot\|_{X}\right)$ be a separable Banach space and let $(\Omega, \mathcal{F}, \mathbb{P})$ be a probability space where $\mathcal{F}$ is the $\sigma$-algebra of measurable subsets of $\Omega$ (called "events") and $\mathbb{P}$ is the probability measure. To connect the state $\omega$ in the probability space $\Omega$ at time 0 with its state after a time of $t$ elapses, we define a flow $\theta=\left\{\theta_{t}\right\}_{t \in \mathbb{R}}$ on $\Omega$ with each $\theta_{t}$ being a mapping $\theta_{t}: \Omega \rightarrow \Omega$ that satisfies

(1) $\theta_{0}=\operatorname{Id}_{\Omega}$

(2) $\theta_{s} \circ \theta_{t}=\theta_{s+t}$ for all $s, t \in \mathbb{R}$,

(3) the mapping $(t, \omega) \mapsto \theta_{t} \omega$ is measurable and

(4) the probability measure $\mathbb{P}$ is preserved by $\theta_{t}$, i.e., $\theta_{t} \mathbb{P}=\mathbb{P}$.

This set-up establishes a time-dependent family $\theta$ that tracks the noise, and $(\Omega, \mathcal{F}, \mathbb{P}, \theta)$ is called a metric dynamical system $[1]$. 
Definition 1 A stochastic process $\{\varphi(t, \omega)\}_{t \geq 0, \omega \in \Omega}$ is said to be a continuous $\operatorname{RDS}$ over $\left(\Omega, \mathcal{F}, \mathbb{P},\left(\theta_{t}\right)_{t \in \mathbb{R}}\right)$ with state space $X$ if $\varphi:[0,+\infty) \times \Omega \times X \rightarrow X$ is $(\mathcal{B}[0,+\infty) \times \mathcal{F} \times \mathcal{B}(X), \mathcal{B}(X))$ - measurable, and for each $\omega \in \Omega$,

(i) the mapping $\varphi(t, \omega): X \rightarrow X, x \mapsto \varphi(t, \omega) x$ is continuous for every $t \geq 0$;

(ii) $\varphi(0, \omega)$ is the identity operator on $X$;

(iii) (cocycle property) $\varphi(t+s, \omega)=\varphi\left(t, \theta_{s} \omega\right) \varphi(s, \omega)$ for all $s, t \geq 0$.

Definition 2 (i) A set-valued mapping $K: \omega \rightarrow 2^{X} \backslash \emptyset$ is said to be a random set if the mapping $\omega \mapsto \operatorname{dist}_{X}(x, K(\omega))$ is measurable for any $x \in X$.

(ii) A random set $K(\omega)$ is said to be bounded if $K(\omega)$ is bounded for a.e. $\omega \in \Omega$; a random set $K(\omega)$ is said to be compact if $K(\omega)$ is compact for a.e. $\omega \in \Omega$; a random set is said to be closed if $K(\omega)$ is closed for a.e. $\omega \in \Omega$.

(iv) A bounded random set $K(\omega) \subset X$ is said to be tempered with respect to $\left(\theta_{t}\right)_{t \in \mathbb{R}}$ if for a.e. $\omega \in \Omega$,

$$
\lim _{t \rightarrow \infty} e^{-\beta t} \sup _{x \in K\left(\theta_{-t} \omega\right)}\|x\|_{X}=0, \quad \text { for all } \beta>0
$$

a random variable $\omega \mapsto r(\omega) \in \mathbb{R}$ is said to be tempered with respect to $\left(\theta_{t}\right)_{t \in \mathbb{R}}$ if for a.e. $\omega \in \Omega$,

$$
\lim _{t \rightarrow \infty} e^{-\beta t} \sup _{t \in \mathbb{R}}\left|r\left(\theta_{-t} \omega\right)\right|=0, \quad \text { for all } \beta>0 .
$$

In what follows we use $\mathcal{D}(X)$ to denote the set of all tempered random sets of $X$.

Definition 3 A random set $\Gamma(\omega) \subset X$ is called a random absorbing set in $\mathcal{D}(X)$ if for any $K \in \mathcal{D}(X)$ and a.e. $\omega \in \Omega$, there exists $T_{K}(\omega)>0$ such that

$$
\varphi\left(t, \theta_{-t} \omega\right) K\left(\theta_{-t} \omega\right) \subset \Gamma(\omega), \quad \forall t \geq T_{K}(\omega) .
$$

Definition 4 Let $\{\varphi(t, \omega)\}_{t \geq 0, \omega \in \Omega}$ be an $\operatorname{RDS}$ over $\left(\Omega, \mathcal{F}, \mathbb{P},\left(\theta_{t}\right)_{t \in \mathbb{R}}\right)$ with state space $X$ and let $\mathcal{A}(\omega)(\subset X)$ be a random set. Then $\mathcal{A}(\omega)$ is called a global random $\mathcal{D}$ attractor (or pullback $\mathcal{D}$ attractor) for $\{\varphi(t, \omega)\}_{t \geq 0, \omega \in \Omega}$ if $\omega \mapsto \mathcal{A}(\omega)$ satisfies

(i) (random compactness) $\mathcal{A}(\omega)$ is a compact set of $X$ for a.e. $\omega \in \Omega$;

(ii) (invariance) for a.e. $\omega \in \Omega$ and all $t \geq 0$, it holds

$$
\varphi(t, \omega) \mathcal{A}(\omega)=\mathcal{A}\left(\theta_{t} \omega\right)
$$

(iii) (attracting property) for any $K \in \mathcal{D}(X)$ and a.e. $\omega \in \Omega$,

$$
\lim _{t \rightarrow \infty} \operatorname{dist}_{X}\left(\varphi\left(t, \theta_{-t} \omega\right) K\left(\theta_{-t} \omega\right), A(\omega)\right)=0,
$$

where

$$
\operatorname{dist}_{X}(G, H)=\sup _{g \in G} \inf _{h \in H}\|g-h\|_{X}
$$

is the Hausdorff semi-metric for $G, H \subseteq X$. 
Proposition 1 [9,13] Let $\Gamma \in \mathcal{D}(X)$ be an absorbing set for the continuous random dynamical system $\{\varphi(t, \omega)\}_{t \geq 0, \omega \in \Omega}$ which is closed and satisfies the asymptotic compactness condition for a.e. $\omega \in \Omega$, i.e., each sequence $x_{n} \in$ $\varphi\left(t_{n}, \theta_{-t_{n}} \omega\right) \Gamma\left(\theta_{-t_{n}} \omega\right)$ has a convergent subsequence in $X$ when $t_{n} \rightarrow \infty$. Then the cocycle $\varphi$ has a unique global random attractor with component subsets

$$
\mathcal{A}(\omega)=\bigcap_{\tau \geq T_{\Gamma}(\omega)} \overline{\bigcup_{t \geq \tau} \varphi\left(t, \theta_{-t} \omega\right) \Gamma\left(\theta_{-t} \omega\right)} .
$$

If the pullback absorbing set is positively invariant, i.e., $\varphi(t, \omega) \Gamma(\omega) \subset \Gamma\left(\theta_{t} \omega\right)$ for all $t \geq 0$, then

$$
\mathcal{A}(\omega)=\bigcap_{t \geq 0} \overline{\varphi\left(t, \theta_{-t} \omega\right) \Gamma\left(\theta_{-t} \omega\right)} .
$$

Remark 1 When the state space $X=\mathbb{R}^{d}$ as in this paper, the asymptotic compactness follows trivially. Note that the random attractor is path-wise attracting in the pullback sense, but does not need to be path-wise attracting in the forward sense, although it is forward attracting in probability, due to some possible large deviations, see e.g., Arnold [1].

\section{Random population model}

In this section we will study the random system $(3)-(5)$. In particular, we assume that the system inputs are perturbed by real noise, i.e., $B_{j}\left(\theta_{t} \omega\right),(j=$ $1,2,3)$ are continuous and essentially bounded:

$$
B_{j}\left(\theta_{t} \omega\right) \in b_{j} \cdot\left[1-\sigma_{j}, 1+\sigma_{j}\right], \quad b_{j}>0, \quad 0<\sigma_{j}<1, \quad j=1,2,3 .
$$

Bounded noise can be modeled in various ways. For example in [2], given a stochastic process $Z_{t}$ such as an Ornstein-Uhlenbeck process, the stochastic process

$$
\zeta\left(Z_{t}\right):=\zeta_{0}\left(1-2 \sigma \frac{Z_{t}}{1+Z_{t}^{2}}\right)
$$

where $\zeta_{0}$ and $\sigma$ are positive constants with $\sigma \in(0,1)$, takes values in the interval $\zeta_{0}[1-\sigma, 1+\sigma]$ and tends to peak around $\zeta_{0}(1 \pm \sigma)$. It is thus suitable for a noisy switching scenario. In another example, the stochastic process

$$
\eta\left(Z_{t}\right):=\eta_{0}\left(1-\frac{2 \sigma}{\pi} \arctan Z_{t}\right)
$$

where $\eta_{0}$ and $\sigma$ are positive constants with $\sigma \in(0,1)$ takes values in the interval $\eta_{0}[1-\sigma, 1+\sigma]$ and is centered at $\eta_{0}$. In the theory of random dynamical systems the driving noise process $Z_{t}(\omega)$ is replaced by a canonical driving system $\theta_{t} \omega$. This simplification allows a better understanding of the path-wise approach to model noise: a system influenced by stochastic processes for each single realization $\omega$ can be interpreted as wandering along a path $\theta_{t} \omega$ in $\Omega$ and thus may provide additional statistical/geological information to the modeler. 
3.1 Properties of solutions

In this subsection we will prove that solutions to equations (3)-(5) exist and are non-negative for any non-negative initial conditions and generate a random dynamical system. For convenience, denote by $u(t)=(x(t), y(t), z(t))^{T}$ and let

$$
\mathbb{R}_{+}^{3}=\left\{(x, y, z) \in \mathbb{R}^{3}: x \geq 0, y \geq 0, z \geq 0\right\} .
$$

Lemma 1 For any $\omega \in \Omega$, any $t_{0} \in \mathbb{R}$, and any initial data $u_{0}:=\left(x\left(t_{0}\right)\right.$, $\left.y\left(t_{0}\right), z\left(t_{0}\right)\right)^{T} \in \mathbb{R}_{+}^{3}$, system (3)-(5) admits a unique non-negative bounded solution $u\left(\cdot ; t_{0}, \omega, u_{0}\right) \in C\left(\left[t_{0}, \infty\right), \mathbb{R}_{+}^{3}\right)$ with $u\left(t_{0} ; t_{0}, \omega, u_{0}\right)=u_{0}$ provided that

$$
m_{1}-m_{2}+a_{21}-a_{12}>0 \text { and } a_{31}-a_{13}>0 .
$$

Moreover the solution generates a random dynamical system $\varphi(t, \omega)(\cdot)$ defined as

$$
\varphi(t, \omega) u_{0}=u\left(t ; 0, \omega, u_{0}\right), \quad \forall t \geq t_{0}, u_{0} \in \mathbb{R}_{+}^{3}, \omega \in \Omega .
$$

Proof With $u(t)=(x(t), y(t), z(t))^{T}$, system (3)-(5) can be written as

$$
\dot{u}(t)=L\left(\theta_{t} \omega\right) \cdot u+f(u),
$$

where

$$
L\left(\theta_{t} \omega\right)=\left(\begin{array}{ccc}
-B_{1}\left(\theta_{t} \omega\right) & 0 & 0 \\
0 & B_{1}\left(\theta_{t} \omega\right) & 0 \\
0 & 0 & B_{3}\left(\theta_{t} \omega\right)
\end{array}\right),
$$

and $f: \mathbb{R}_{+}^{3} \rightarrow \mathbb{R}^{3}$ is given by

$$
f(u)=\left(\begin{array}{c}
a_{12} x y+a_{13} x z \\
-\left(a_{21}+m_{1}\right) x y-a_{22} y^{2}-a_{23} y z \\
m_{2} x y-a_{31} x z-a_{32} y z-a_{33} z^{3}
\end{array}\right) .
$$

Since each $B_{j}\left(\theta_{t} \omega\right)$ is bounded, operator $L$ generates an evolution system on $\mathbb{R}^{3}$. In addition, function $f$ is continuously differentiable and locally Lipschitz in $\mathbb{R}^{3}$. Hence system (3)-(5) possesses a unique local solution. For $u(t) \in \mathbb{R}_{+}^{3}$, define $\|u(t)\|_{1}=x(t)+y(t)+z(t):=S(t)$. Then the time derivative of $S(t)$ along solutions to (3)-(5) satisfies

$$
\begin{aligned}
\frac{\mathrm{d} S(t)}{\mathrm{d} t}= & -B_{1}\left(\theta_{t} \omega\right) x+B_{2}\left(\theta_{t} \omega\right) y+B_{3}\left(\theta_{t} \omega\right) z-a_{22} y^{2}-a_{33} z^{2}-\left(a_{23}+a_{32}\right) y z \\
& -\left(m_{1}-m_{2}+a_{21}-a_{12}\right) x y-\left(a_{31}-a_{13}\right) x z \\
\leq & -b_{1}\left(1-\sigma_{1}\right) x+b_{2}\left(1+\sigma_{2}\right) y+b_{3}\left(1+\sigma_{3}\right) z-a(y+z)(x+y+z) \\
\leq & (y+z)[b-a S]
\end{aligned}
$$

where

$$
\begin{aligned}
a & :=\min \left\{a_{22}, a_{33}, a_{23}+a_{32}, m_{1}-m_{2}+a_{21}-a_{12}, a_{31}-a_{13}\right\} \\
b & :=\max \left\{b_{2}\left(1+\sigma_{2}\right), b_{3}\left(1+\sigma_{3}\right)\right\} .
\end{aligned}
$$


For any $S\left(t_{0}\right) \geq b / a, S(t)$ will be non-increasing as long as it stays in the region $\{S: S \geq b / a\}$. If it ever enters the region $\{S: S<b / a\}$, then we have $y+z \leq S<b / a$ and hence

$$
\frac{\mathrm{d} S(t)}{\mathrm{d} t}<\frac{b}{a} \cdot(b-a S)
$$

This implies that

$$
S(t) \leq \frac{b}{a}+S\left(t_{0}\right) e^{-b t} \leq \frac{b}{a}+S\left(t_{0}\right) e^{-b t_{0}} \text { for any } t \geq t_{0},
$$

and hence $\|u(t)\|_{1}$ is bounded and the local solution can be extended to a global solution $u\left(\cdot ; t_{0}, \omega, u_{0}\right) \in C^{1}\left(\left[t_{0}, \infty\right), \mathbb{R}^{3}\right)$.

It is straightforward to check the cocycle property

$$
u\left(t+t_{0} ; t_{0}, \omega, u_{0}\right)=u\left(t ; 0, \theta_{t_{0}} \omega, u_{0}\right)
$$

for all $t_{0} \in \mathbb{R}, t \geq t_{0}, \omega \in \Omega, u_{0} \in \mathbb{R}_{+}^{3}$. This allows us to define a mapping $\varphi(t, \omega)(\cdot)$, which will be our random dynamical system, as

$$
\varphi(t, \omega) u_{0}=u\left(t ; 0, \omega, u_{0}\right), \quad \forall t \geq 0, u_{0} \in \mathbb{R}_{+}^{3}, \omega \in \Omega
$$

From now on, we will simply write $u\left(t ; \omega, u_{0}\right)$ instead of $u\left(t ; 0, \omega, u_{0}\right)$. Also in what follows, when $\omega \in \Omega$ fixed, we will not mention explicitly the random parameter and will write $u\left(t ; \omega, u_{0}\right)$ as $u(t)$ in short.

By continuity of solutions, each solution has to take value 0 before it reaches a negative value. (1) With $x=0$, equation (3) becomes $\dot{x}(t)=0$ which implies that $x$ will vanish once it reaches 0 . (2) With $y=0$, (4) becomes $\dot{y}(t)=0$ which implies that $y$ will also vanish once it reaches 0 . (3) With $z=0$, (5) becomes $\dot{z}(t)=m_{2} x y \geq 0$ for $x, y \geq 0$. Hence $u(t) \in \mathbb{R}_{+}^{3}$ for any $u_{0} \in \mathbb{R}_{+}^{3}$, i.e., $\mathbb{R}_{+}^{3}$ is positively invariant.

For any $u_{0} \in \mathbb{R}_{+}^{3}$, solution $u\left(\cdot ; \omega, u_{0}\right) \in \mathbb{R}_{+}^{3}$ for $t \in[0, \infty)$. Since function $f(u)$ is continuous in $u, u:[0, \infty) \times \Omega \times \mathbb{R}_{+}^{3} \rightarrow \mathbb{R}_{+}^{3},\left(t ; \omega, u_{0}\right) \mapsto u\left(t ; \omega, u_{0}\right)$ is $\left(\mathcal{B}[0, \infty) \times \mathcal{F}_{0} \times \mathcal{B}\left(\mathbb{R}_{+}^{3}\right), \mathcal{B}\left(\mathbb{R}_{+}^{3}\right)\right)$-measurable. It then follows directly that (3)-(5) generate a continuous random dynamical system $\varphi(t, \omega)(\cdot)$ defined by (18).

3.2 Existence of a unique global random attractor

In this subsection we will prove that the random dynamical system (RDS) $\varphi(t, \omega)(\cdot)$ possesses a random attractor. To this end, we will first prove in the next lemma that the $\operatorname{RDS} \varphi(t, \omega)(\cdot)$ has a tempered random bounded absorbing set. 
Lemma 2 Assume that $m_{1}-m_{2}+a_{21}-a_{12}>0$ and $a_{31}-a_{13}>0$. Then for each $\omega \in \Omega$, there exists a tempered bounded closed random absorbing set $\Gamma(\omega) \in \mathcal{D}\left(\mathbb{R}_{+}^{3}\right)$ of the random dynamical system $\{\varphi(t, \omega)\}_{t \geq 0, \omega \in \Omega}$ such that for any $K \in \mathcal{D}\left(\mathbb{R}_{+}^{3}\right)$ and each $\omega \in \Omega$, there exists $T_{K}(\omega)>0$ yielding

$$
\varphi\left(t, \theta_{-t} \omega\right) K\left(\theta_{-t} \omega\right) \subset \Gamma(\omega), \quad \forall t \geq T_{K}(\omega) .
$$

More precisely, for a given $\varepsilon>0$, the set $\Gamma(\omega)$ can be chosen as the deterministic set

$$
\Gamma_{\varepsilon}:=\left\{(x, y, z) \in \mathbb{R}_{+}^{3}: x+y+z \leq \frac{b}{a}+\varepsilon\right\}
$$

for all $\omega \in \Omega$, i.e. $\Gamma(\omega)=\Gamma_{\varepsilon}$ for all $\omega \in \Omega$.

Proof For any $\varepsilon>0$, define the set

$$
\Gamma_{\varepsilon}:=\left\{(x, y, z) \in \mathbb{R}_{+}^{3}: x+y+z \leq \frac{b}{a}+\varepsilon\right\},
$$

where $a$ and $b$ are as defined in (15) and (16), respectively.

We first prove that $\Gamma_{\varepsilon}$ is invariant.

(a) For any solution of (3) - (5) starting from a point inside $\Gamma_{0}:=\{(x, y, z)$ $\left.\in \mathbb{R}_{+}^{3}: x+y+z \leq b / a\right\}$, since $\mathbb{R}_{+}^{3}$ is positively invariant and $\dot{S}(t) \leq 0$ on $x+y+z=b / a$ according to (17), we have $S(t) \leq b / a$ for all $t \geq t_{0}$. This implies the positive invariance of $\Gamma_{0}$. In addition, it follows from inequality (17) that any $S(t) \in \Gamma_{0}$ satisfies

$$
\dot{S}(t) \leq S(b-a S)
$$

Integrating the Bernoulli type inequality (19) gives

$$
S(t) \leq \frac{b S\left(t_{0}\right)}{a S\left(t_{0}\right)+\left(b-a S\left(t_{0}\right)\right) e^{-b\left(t-t_{0}\right)}},
$$

which implies that

$$
\lim _{t \rightarrow \infty} S(t) \leq \frac{b}{a}, \quad \text { and } \quad \lim _{t_{0} \rightarrow-\infty} S(t) \leq \frac{b}{a}
$$

(b) For any solution starting from a point inside $\Gamma_{\varepsilon} \backslash \Gamma_{0}$, Eq. (19) implies that $\dot{S}(t) \leq 0$ and thus the solution cannot escape from $\Gamma_{\varepsilon}$.

(a) and (b) together indicate that $\Gamma_{\varepsilon}$ is positively invariant. It then remains to show that $\Gamma_{\varepsilon}$ is absorbing.

(c) Recall that $u\left(t ; \omega, u_{0}\right)=\varphi(t, \omega) u_{0}$ denotes the solution of system (3) - (5) satisfying $u\left(0 ; \omega, u_{0}\right)=u_{0}$. Then for any $u_{0}:=u_{0}\left(\theta_{-t} \omega\right) \in K\left(\theta_{-t} \omega\right)$,

$$
\left\|\varphi\left(t, \theta_{-t} \omega\right) u_{0}\right\|_{1}=\| u\left(t ; \theta_{-t} \omega, u_{0}\left(\theta_{-t} \omega\right) \|_{1} \leq S\left(t ; \theta_{-t} \omega, S_{0}\left(\theta_{-t} \omega\right)\right) .\right.
$$


Since we have already proved the positive invariance of $\Gamma_{\varepsilon}$, we only need to check the situation where $S(t) \geq b / a+\varepsilon$. Following a similar procedure to (14), we obtain

$$
\begin{aligned}
\dot{S} & \leq-b_{1}\left(1-\sigma_{1}\right) x+(y+z)[b-a S] \leq-b_{1}\left(1-\sigma_{1}\right) x-a \varepsilon(y+z) \\
& \leq-\min \left\{b_{1}\left(1-\sigma_{1}\right), a \varepsilon\right\} \cdot S,
\end{aligned}
$$

which can be integrated to obtain

$$
S(t, \omega) \leq S_{0} e^{-\min \left\{b_{1}\left(1-\sigma_{1}\right), a \varepsilon\right\} \cdot\left(t-t_{0}\right)} .
$$

Substituting $\omega$ by $\theta_{-t} \omega$ in $(21)$ gives

$$
S\left(t ; \theta_{-t} \omega, S_{0}\left(\theta_{-t} \omega\right)\right) \leq \sup _{u \in K\left(\theta_{-t} \omega\right)}\|u\| \cdot e^{-\min \left\{b_{1}\left(1-\sigma_{1}\right), a \varepsilon\right\} \cdot\left(t-t_{0}\right)} .
$$

Collecting (a), (b) and (c) we conclude that there exists a time $T_{K}(\omega)$ such that when $t>T_{K}, \varphi\left(t, \theta_{-t} \omega\right) u_{0} \in \Gamma_{\varepsilon}$ for all $u_{0} \in K\left(\theta_{-t} \omega\right)$, i.e., $\Gamma_{\varepsilon}$ is a compact absorbing set for any $\varepsilon>0$ and absorbs all bounded sets of $\mathbb{R}_{+}^{3}$.

Lemma 1, Lemma 2 together with Proposition 1 and Remark 1 imply immediately the following Theorem.

Theorem 1 The random dynamical system generated by system (3) - (5) possesses a global random attractor provided that

$$
m_{1}-m_{2}+a_{21}-a_{12}>0 \text { and } a_{31}-a_{13}>0 .
$$

\subsection{Coexistence analysis}

In this subsection we will discuss the coexistence of the three species in random environments. These results provide indirectly information about geometric structures of the random attractor.

To simplify notations, we first define the following quantities:

$$
\begin{aligned}
a_{M} & :=\max \left\{a_{21}-a_{12}, a_{31}-a_{13}, a_{22}, a_{23}, a_{32}, a_{33}\right\} \\
\alpha & :=\max \left\{2 a_{M}, 2 a_{M}-m_{2}+m_{1}\right\} \\
b_{m} & :=\min \left\{b_{2}, b_{3}\right\} \\
\lambda & :=\left(1-\max \left\{\sigma_{2}, \sigma_{3}\right\}\right) b_{m}
\end{aligned}
$$

Theorem 2 The compact set

$$
\Sigma:=\left\{(x, y, z) \in R_{+}^{3}: \quad y+z \geq \frac{\lambda}{\alpha}, \quad x+y+z \leq \frac{b}{a}\right\},
$$

is a positively invariant set provided that

$$
\frac{b_{1}\left(1+\sigma_{1}\right)}{a_{M}} \leq \frac{\lambda}{\alpha}
$$


Proof Summing equations (3) through (5), we obtain

$$
\begin{aligned}
\dot{S}=- & B_{1}\left(\theta_{t} w\right) x+B_{2}\left(\theta_{t} w\right) y+B_{3}\left(\theta_{t} w\right) z+\left(m_{2}-m_{1}\right) x y \\
& -\left[\left(a_{21}-a_{12}\right) x y+\left(a_{31}-a_{13}\right) x z+a_{22} y^{2}+\left(a_{23}+a_{32}\right) y z+a_{33} z^{2}\right] \\
\geq- & b_{1}\left(1+\sigma_{1}\right) x+\left(1-\max \left\{\sigma_{2}, \sigma_{3}\right\}\right) b_{m}(y+z) \\
& +\left(m_{2}-m_{1}\right) x y-a_{M}(y+z) S .
\end{aligned}
$$

If $m_{2}-m_{1}>0$ we have

$$
\begin{aligned}
\dot{S} \geq- & b_{1}\left(1+\sigma_{1}\right) S+\left(1-\max \left\{\sigma_{2}, \sigma_{3}\right\}\right) b_{m}(y+z) \\
& \quad+a_{M}(y+z) S-2 a_{M}(y+z) S \\
= & S\left[a_{M}(y+z)-b_{1}\left(1+\sigma_{1}\right)\right]+(y+z)\left[\left(1-\max \left\{\sigma_{2}, \sigma_{3}\right\}\right) b_{m}-2 a_{M} S\right] .
\end{aligned}
$$

On the other hand, for $m_{2}-m_{1}<0$ we have

$$
\begin{aligned}
\dot{S} \geq & -b_{1}\left(1+\sigma_{1}\right) S+\left(1-\max \left\{\sigma_{2}, \sigma_{3}\right\}\right) b_{m}(y+z) \\
& \quad-a_{M}(y+z) S+\left(m_{2}-m_{1}\right)(y+z) S \\
= & S\left[a_{M}(y+z)-b_{1}\left(1+\sigma_{1}\right)\right] \\
& +(y+z)\left[\left(1-\max \left\{\sigma_{2}, \sigma_{3}\right\}\right) b_{m}-\left(2 a_{M}-m_{2}+m_{1}\right) S\right] .
\end{aligned}
$$

Hence, in summary, we deduce

$$
\dot{S} \geq S\left[a_{M}(y+z)-b_{1}\left(1+\sigma_{1}\right)\right]+(y+z)[\lambda-\alpha S] .
$$

The set $\Sigma$ is nonempty since $\frac{\lambda}{\alpha} \leq \frac{b}{a}$. Moreover, using $\frac{b_{1}\left(1+\sigma_{1}\right)}{a_{M}} \leq \frac{\lambda}{\alpha}$ we arrive at

$$
\dot{S} \geq \frac{b_{1}\left(1+\sigma_{1}\right)}{a_{M}}(\lambda-\alpha S)
$$

by which we complete the proof.

Note that in order for the set $\Sigma$ to be nonempty, the density of predator, $x$ must be smaller than the quantity

$$
\frac{b}{a}-\frac{\lambda}{\alpha}
$$

which implies that the predator plays a crucial role in the coexistence of the three species. This motivates the investigation on conditions to avoid the extinction of the predator.

By using Theorem 2 and equation (3) we have

$$
\dot{x} \geq x\left[-b_{i}\left(\sigma_{1}+1\right)+\min \left\{a_{12}, a_{13}\right\} \frac{\lambda}{\alpha}\right],
$$

then if

$$
\min \left\{a_{12}, a_{13}\right\} \cdot \lambda>\alpha \cdot b_{i}\left(\sigma_{1}+1\right)
$$


the predator never goes to extinction. Hence assuming (24) and (23) the predator and at least one of the preys persist.

To obtain more insight for the coexistence of the two preys, we first suppose that $z=0$, then equation (5) of the system becomes

$$
\dot{z}=m_{2} x y>0
$$

which implies that the prey $z$ never goes to extinction, i.e., the system is persistent. The only possibility against persistence of the system is represented by the extinction of the prey $y$. In this case since $y=0$ is an invariant set the system (3) - (5) becomes

$$
\begin{aligned}
& \dot{x}(t, \omega)=x(t)\left(-B_{1}\left(\theta_{t} \omega\right)+a_{13} z(t)\right), \\
& \dot{z}(t, \omega)=z(t)\left(B_{3}\left(\theta_{t} \omega\right)-a_{31} x(t)-a_{33} z(t)\right),
\end{aligned}
$$

and the attractor of this systems falls into the region:

$$
0<x \leq \frac{b}{a}-\frac{\lambda}{\alpha}, \quad z \geq \frac{\lambda}{\alpha}
$$

Summarily, a sufficient condition for the persistence of the whole system should preclude the instability of the attractor on $y=0$. Hence we impose an easier condition to avoid the convergence to a set on the plane $y=0$. Noticing that equation (4) implies

$$
\dot{y}(t, \omega) \geq y(t)\left[b_{2}\left(1-\sigma_{2}\right)-\left(a_{21}+m_{1}\right)\left(\frac{b}{a}-\frac{\lambda}{\alpha}\right)-a_{22} y(t)-a_{23} \frac{b}{a}\right],
$$

then if $a_{21}, m_{1}$ and $a_{23}$ are sufficiently small to ensure

$$
b_{2}\left(1-\sigma_{2}\right)-\left(a_{21}+m_{1}\right)\left(\frac{b}{a}-\frac{\lambda}{\alpha}\right)-a_{23} \frac{b}{a}>0
$$

we obtain that $y$ never goes to extinction and the system is persistent.

\section{Stochastic population model}

In this section we will investigate the stochastic system (6) - (8). To this end, we first transform stochastic equations $(6),(7)$ and (8) into random equations with random coefficients and without white noise.

Let $\Omega$ be defined by

$$
\Omega=\{\omega \in C(\mathbb{R}, \mathbb{R}): \omega(0)=0\}=C_{0}(\mathbb{R}, \mathbb{R}),
$$

$\mathcal{F}$ be the Borel $\sigma$-algebra on $\Omega$ generated by the compact open topology [1], and $\mathbb{P}$ be the corresponding Wiener measure on $\mathcal{F}$. Letting

$$
\theta_{t} \omega(\cdot)=\omega(\cdot+t)-\omega(t)
$$


$\left(\Omega, \mathcal{F}, \mathbb{P},\left(\theta_{t}\right)_{t \in \mathbb{R}}\right)$ is a metric dynamical system. Now introduce the following Ornstein-Uhlenbeck process on $\left(\Omega, \mathcal{F}, \mathbb{P},\left(\theta_{t}\right)_{t \in \mathbb{R}}\right)$

$$
\delta\left(\theta_{t} \omega\right)=-\int_{-\infty}^{0} e^{s} \theta_{t} \omega(s) \mathrm{d} s, \quad t \in \mathbb{R}, \quad \omega \in \Omega_{0} .
$$

which solves the following Ornstein-Uhlenbeck equation $[1,8]$

$$
\mathrm{d} \delta+\delta \mathrm{d} t=\mathrm{d} W(t), \quad t \in \mathbb{R},
$$

where $W(t)(\omega)=W(t, \omega)=\omega(t)$ for $\omega \in \Omega, t \in \mathbb{R}$, is a two sided Wiener process.

Proposition $2([1,8])$ There exists a $\theta_{t}$-invariant set $\widetilde{\Omega} \in \mathcal{F}$ of $\Omega$ of full $\mathbb{P}$ measure such that for $\omega \in \widetilde{\Omega}$, we have

(i) the random variable $|\delta(\omega)|$ is tempered, i.e., for $\omega \in \widetilde{\Omega}$,

$$
\lim _{t \rightarrow+\infty} e^{-\beta t} \sup _{t \in \mathbb{R}}\left|\delta\left(\theta_{-t} \omega\right)\right|=0, \quad \forall \beta>0
$$

(ii) the mapping

$$
(t, \omega) \rightarrow \delta\left(\theta_{t} \omega\right)=-\int_{-\infty}^{0} e^{s} \omega(t+s) \mathrm{d} s+\omega(t)
$$

is a stationary solution of Ornstein-Uhlenbeck equation (26) with continuous trajectories;

(iii) In addition, for any $\omega \in \tilde{\Omega}$ :

$$
\begin{aligned}
\lim _{t \rightarrow \pm \infty} \frac{\left|\delta\left(\theta_{t} \omega\right)\right|}{t} & =0 ; \\
\lim _{t \rightarrow \pm \infty} \frac{1}{t} \int_{0}^{t} \delta\left(\theta_{s} \omega\right) d s & =0 \\
\lim _{t \rightarrow \pm \infty} \frac{1}{t} \int_{0}^{t}\left|\delta\left(\theta_{s} \omega\right)\right| d s & =\mathbb{E}[\delta]<\infty .
\end{aligned}
$$

In what follows we will consider $\theta$ defined in $(25)$ on $\tilde{\Omega}$ instead of $\Omega$. This mapping has the same properties as the original one if we choose for $\mathcal{F}$ the trace $\sigma$-algebra with respect to $\tilde{\Omega}$ also by $\mathcal{F}$. For simplicity, we will still denote $\tilde{\Omega}$ by $\Omega$ when the content is clear. 
4.1 From stochastic system to random system

To get quantitative analysis of (6) - (8), we first transform them into random ordinary differential equations with random parameters, but with no white noise, by performing the change of variables

$$
\tilde{X}=X \cdot e^{-\mu \delta\left(\theta_{t} \omega\right)}, \quad \tilde{Y}=Y \cdot e^{-\mu \delta\left(\theta_{t} \omega\right)}, \quad \tilde{Z}=Z \cdot e^{-\mu \delta\left(\theta_{t} \omega\right)},
$$

equations $(6)-(8)$ become (with $\delta\left(\theta_{t} \omega\right)$ written in short as $\delta$ )

$$
\begin{aligned}
\frac{\mathrm{d} \tilde{X}}{\mathrm{~d} t} & =\tilde{X}\left[-b_{1}+\mu \delta+e^{\mu \delta}\left(a_{12} \tilde{Y}+a_{13} \tilde{Z}\right)\right], \\
\frac{\mathrm{d} \tilde{Y}}{\mathrm{~d} t} & =\tilde{Y}\left[b_{2}+\mu \delta-e^{\mu \delta}\left(\left(a_{21}+m_{1}\right) \tilde{X}+a_{22} \tilde{Y}+a_{23} \tilde{Z}\right)\right], \\
\frac{\mathrm{d} \tilde{Z}}{\mathrm{~d} t} & =\tilde{Z}\left[b_{3}+\mu \delta-e^{\mu \delta}\left(a_{31} \tilde{X}+a_{32} \tilde{Y}+a_{33} \tilde{Z}\right)\right]+m_{2} e^{\mu \delta} \tilde{X} \tilde{Y} .
\end{aligned}
$$

Notice that (30)-(32) is a random system of ordinary differential equations but the main difference with the system (3)-(5) is that the random terms in the latter are unbounded, what makes necessary the use of the properties of the Orstein-Uhlenbeck process established in Proposition 2.

For simplicity denote by

$$
v(t, \omega):=(\tilde{X}(t, \omega), \tilde{Y}(t, \omega), \tilde{Z}(t, \omega))^{T},
$$

and rewrite $(30)-(32)$ as

$$
\frac{\mathrm{d} v}{\mathrm{~d} t}=\left(\begin{array}{ccc}
-b_{1}+\mu \delta & 0 & 0 \\
0 & b_{2}+\mu \delta & 0 \\
0 & 0 & b_{3}+\mu \delta
\end{array}\right) v+g\left(v, \theta_{t} \omega\right),
$$

where

$$
g\left(v, \theta_{t} \omega\right)=e^{\mu \delta\left(\theta_{t} \omega\right)}\left(\begin{array}{c}
a_{12} \tilde{X} \tilde{Y}+a_{13} \tilde{X} \tilde{Z} \\
-\left(a_{21}+m_{1}\right) \tilde{X} \tilde{Y}-a_{22} \tilde{Y}{ }^{2}-a_{23} \tilde{Y} \tilde{Z} \\
m_{2} \tilde{X} \tilde{Y}-a_{31} \tilde{X} \tilde{Z}-a_{32} \tilde{Y} \tilde{Z}-a_{33} \tilde{Z}^{2}
\end{array}\right)
$$

First observe that $\mathbb{R}_{+}^{3}$ is positively invariant. In fact, since

$$
\left.\frac{\mathrm{d} \tilde{X}}{\mathrm{~d} t}\right|_{\tilde{X}=0}=0,\left.\quad \frac{\mathrm{d} \tilde{Y}}{\mathrm{~d} t}\right|_{\tilde{Y}=0}=0,
$$

the plane $\tilde{X}=0$ and the plane $\tilde{Y}=0$ are invariant. In addition

$$
\left.\frac{\mathrm{d} \tilde{Z}}{d t}\right|_{\tilde{Z}=0}=m_{2} \tilde{X} \tilde{Y} \geq 0 \quad \text { for all } \tilde{X}, \tilde{Y} \geq 0
$$

on the plane $\tilde{Z}=0$. 
4.2 Properties of solutions

In this subsection we show that for any initial condition $v(0)=v_{0} \in \mathbb{R}_{+}^{3}$, solutions to (33) and equivalently to (30) - (32) exist and generate a random dynamical system.

Lemma 3 Assume that

$$
m_{1}-m_{2}+a_{21}-a_{12}>0 \text { and } a_{31}-a_{13}>0 .
$$

Let $T>0$ and $v_{0} \in \mathbb{R}_{+}^{3}$ be fixed. Then for every $\omega \in \Omega$, system (33) admits a unique solution $v\left(\cdot, \omega, v_{0}\right) \in C\left([0, T], \mathbb{R}_{+}^{3}\right)$ and the solution generates a random dynamical system $\psi(t, \omega)(\cdot)$ defined by

$$
\psi(t, \omega) v_{0}=v\left(t ; 0, \omega, v_{0}\right), \quad \forall t \geq 0, v_{0} \in \mathbb{R}_{+}^{3}, \omega \in \Omega .
$$

Moreover, defining $\eta$ by

$$
\eta\left(t ; \omega, v_{0}\right)=e^{\mu \delta\left(\theta_{t} \omega\right)} \psi\left(t ; \omega, e^{-\mu \delta\left(\theta_{t} \omega\right)} v_{0}\right), \quad \forall t \geq 0, v_{0} \in \mathbb{R}_{+}^{3}, \omega \in \Omega,
$$

then $\eta$ is another random dynamical system for which the process

$$
(\omega, t) \mapsto \eta\left(t ; \omega, v_{0}\right)
$$

solves the original system (6) - (8) for any initial condition $v_{0} \in \mathbb{R}_{+}^{3}$.

Proof For any fixed $T>0$, notice that function $g(t, v)$ is continuous in $t$ and locally Lipschitz in $v$, with a uniformly bounded Lipschitz constant on $[0, T]$, hence by classical results from the theory of ordinary differential equations, system $(33)$ has a local solution $v\left(\cdot, \omega, v_{0}\right) \in C\left(\left[0, T_{\max }\right), \mathbb{R}^{3}\right)$ where $\left[0, T_{\max }\right)$ is the maximal interval of existence of the solution to (33). Define

$$
\|v(t)\|:=\tilde{X}+\tilde{Y}+\tilde{Z}
$$

then from (30) - (32) it follows that

$$
\begin{aligned}
\frac{\mathrm{d}}{\mathrm{d} t}\|v\|= & -b_{1} \tilde{X}+b_{2} \tilde{Y}+b_{3} \tilde{Z}+\mu \delta(\tilde{X}+\tilde{Y}+\tilde{Z})-e^{\mu \delta}\left[a_{22} \tilde{Y}^{2}+\left(a_{23}+a_{32}\right) \tilde{Y} \tilde{Z}\right. \\
& \left.+\left(a_{12}-a_{21}+m_{2}-m_{1}\right) \tilde{X} \tilde{Y}+\left(a_{31}-a_{13}\right) \tilde{X} \tilde{Z}+a_{33} \tilde{Z}^{2}\right] \\
\leq & -b_{1}\|v\|+\tilde{b}(y+z)+\mu \delta\|v\|-a e^{\mu \delta}(y+z)\|v\|, \\
= & \left(-b_{1}+\mu \delta\right)\|v\|+(y+z)\left(\tilde{b}-a e^{\mu \delta}\|v\|\right),
\end{aligned}
$$

where $a$ is as defined in (15) and

$$
\tilde{b}:=\max \left\{b_{1}+b_{2}, b_{1}+b_{3}\right\} .
$$

We next discuss the inequality (34) case by case.

(1) When $a e^{\mu \delta}\|v\| \geq \tilde{b}$, we have

$$
\frac{\mathrm{d}}{\mathrm{d} t}\|v\| \leq\left(-b_{1}+\mu \delta\right)\|v\|
$$


which implies that

$$
\|v(t)\| \leq\left\|v_{0}\right\| e^{-b_{1} t+\mu \int_{0}^{t} \delta\left(\theta_{s} \omega\right) d s} \leq\|v(0)\| e^{\mu \int_{0}^{t}\left|\delta\left(\theta_{s} \omega\right)\right| d s} .
$$

(2) When $a e^{\mu \delta}\|v\|<\tilde{b}$, we have $y+z \leq\|v\|<\frac{\tilde{b}}{a} e^{-\mu \delta}$ and consequently

$$
\frac{\mathrm{d}}{\mathrm{d} t}\|v\| \leq\left(-b_{1}-\tilde{b}+\mu \delta\right)\|v\|+\frac{\tilde{b}^{2}}{a} e^{-\mu \delta} .
$$

Integrating (36) gives

$$
\begin{aligned}
\|v\| \leq & \left\|v_{0}\right\| e^{-\left(b_{1}+b\right) t+\mu \int_{0}^{t} \delta\left(\theta_{s} \omega\right) d s} \\
& +\frac{\tilde{b}^{2}}{a} e^{-\left(b_{1}+b\right) t+\mu \int_{0}^{t} \delta\left(\theta_{s} \omega\right) d s} \int_{0}^{t} e^{\left(b_{1}+\tilde{b}\right) s-\mu \delta\left(\theta_{s} \omega\right)-\mu \int_{0}^{s} \delta\left(\theta_{\tau} \omega\right) d \tau} d s \\
\leq & \left\|v_{0}\right\| e^{\mu \int_{0}^{t}\left|\delta\left(\theta_{s} \omega\right)\right| d s}+\gamma(\omega)
\end{aligned}
$$

where

$\gamma(\omega):=\frac{\tilde{b}^{2}}{a} \cdot \max _{t \in[0, T]}\left\{e^{-\left(b_{1}+b\right) t+\mu \int_{0}^{t} \delta\left(\theta_{s} \omega\right) d s} \int_{0}^{t} e^{\left(b_{1}+\tilde{b}\right) s-\mu \delta\left(\theta_{s} \omega\right)-\mu \int_{0}^{s} \delta\left(\theta_{\tau} \omega\right) d \tau} d s\right\}$.

In summary, the solution $v$ is defined on any interval $[0, T]$.

Due to properties of $\delta\left(\theta_{t} \omega\right)$, function $g\left(u, \theta_{t} \omega\right)$ is continuous in $v, t$ and measurable in $\omega$, then for any $T>0, v:[0, T] \times \Omega \times \mathbb{R}_{+}^{3} \rightarrow \mathbb{R}_{+}^{3},\left(t ; \omega, v_{0}\right) \mapsto$ $v\left(t ; \omega, v_{0}\right)$ is $\left(\mathcal{B}[0, T] \times \mathcal{F} \times \mathcal{B}\left(\mathbb{R}_{+}^{3}\right), \mathcal{B}\left(\mathbb{R}_{+}^{3}\right)\right)$-measurable, and hence (33) generates a random dynamical system $\psi(t, \omega)(\cdot)$ defined by

$$
\psi(t, \omega) v_{0}=v\left(t ; 0, \omega, v_{0}\right), \quad t \geq 0, v_{0} \in \mathbb{R}_{+}^{3}, \omega \in \Omega .
$$

It remains to show that $\eta$ and $\psi$ are conjugated random dynamical systems. In fact by the chain rule we have

$$
\begin{aligned}
\mathrm{d} \eta(t)= & \mathrm{d}\left(e^{\mu \delta\left(\theta_{t} \omega\right)} \psi\right)=e^{\mu \delta\left(\theta_{t} \omega\right)} \mathrm{d} \psi+\mu e^{\mu \delta\left(\theta_{t} \omega\right)} \psi \circ \mathrm{d} \delta\left(\theta_{t} \omega\right) \\
= & {\left[\left(\begin{array}{ccc}
-b_{1}+\mu \delta & 0 & 0 \\
0 & b_{2}+\mu \delta & 0 \\
0 & 0 & b_{3}+\mu \delta\left(\theta_{t} \omega\right)
\end{array}\right) \psi+g(\psi)\right] e^{\mu \delta\left(\theta_{t} \omega\right)} \mathrm{d} t } \\
& +\left(-\mu \delta\left(\theta_{t} \omega\right)\right) e^{\mu \delta\left(\theta_{t} \omega\right)} \psi \mathrm{d} t+\mu e^{\mu \delta\left(\theta_{t} \omega\right)} \psi \circ \mathrm{d} W(t) \\
= & {\left[\left(\begin{array}{ccc}
-b_{1} & 0 & 0 \\
0 & b_{2} & 0 \\
0 & 0 & b_{3}
\end{array}\right) \eta+g(\eta)\right] \mathrm{d} t+\mu \eta \circ \mathrm{d} W(t) . }
\end{aligned}
$$

The proof is complete. 
4.3 Existence of a unique global random attractor

In this subsection we prove that the random dynamical system $\{\eta(t, \omega)\}_{t>0, \omega \in \Omega}$ generated by (6) - (8) has a unique global random attractor. To this end, we first prove that there exists a closed random tempered absorbing set for the $\operatorname{RDS}\{\eta(t, \omega)\}_{t \geq 0, \omega \in \Omega}$.

Lemma 4 Assume that

$$
m_{1}-m_{2}+a_{21}-a_{12}>0 \text { and } a_{31}-a_{13}>0 .
$$

Then there exists a closed random tempered set $\Gamma \in \mathcal{D}$ such that for all $K \in \mathcal{D}$ and a. e. $\omega \in \Omega$, there exists $T_{K}(\omega)>0$ such that

$$
\eta\left(t, \theta_{-t} \omega, K\left(\theta_{-t} \omega\right)\right) \subset \Gamma(\omega), \quad \forall t>T_{K}(\omega) .
$$

Proof According to (35) and (37), for any $v_{0} \in \mathbb{R}_{+}^{3}$,

$$
\begin{aligned}
\|v(t)\| \leq & \max \left\{\left\|v_{0}\right\| e^{-b_{1} t+\mu \int_{0}^{t} \delta\left(\theta_{s} \omega\right) d s},\left\|v_{0}\right\| e^{-\left(b_{1}+b\right) t+\mu \int_{0}^{t} \delta\left(\theta_{s} \omega\right) d s}\right. \\
& \left.+\frac{\tilde{b}^{2}}{a} e^{-\left(b_{1}+b\right) t+\mu \int_{0}^{t} \delta\left(\theta_{s} \omega\right) d s} \int_{0}^{t} e^{\left(b_{1}+\tilde{b}\right) s-\mu \delta\left(\theta_{s} \omega\right)-\mu \int_{0}^{s} \delta\left(\theta_{\tau} \omega\right) d \tau} d s\right\} \\
\leq & \left\|v_{0}\right\| e^{-b_{1} t+\mu \int_{0}^{t} \delta\left(\theta_{s} \omega\right) d s} \\
& +\frac{\tilde{b}^{2}}{a} e^{-\left(b_{1}+b\right) t+\mu \int_{0}^{t} \delta\left(\theta_{s} \omega\right) d s} \int_{0}^{t} e^{\left(b_{1}+\tilde{b}\right) s-\mu \delta\left(\theta_{s} \omega\right)-\mu \int_{0}^{s} \delta\left(\theta_{\tau} \omega\right) d \tau} d s
\end{aligned}
$$

Replacing $\omega$ by $\theta_{-t} \omega$ and $v_{0}$ by $e^{-\mu \delta\left(\theta_{-t} \omega\right)} v_{0}$ in the expression of $\psi$ gives

$$
\begin{aligned}
& \| \psi\left(t, \theta_{-t} \omega, e^{-\mu \delta\left(\theta_{-t} \omega\right)} v_{0} \|\right. \\
& \leq\left\|e^{-\mu \delta\left(\theta_{-t} \omega\right)} v_{0}\right\| e^{-b_{1} t+\mu \int_{0}^{t} \delta\left(\theta_{s-t} \omega\right) d s} \\
& +\frac{\tilde{b}^{2}}{a} e^{-\left(b_{1}+b\right) t+\mu \int_{0}^{t} \delta\left(\theta_{s-t} \omega\right) d s} \int_{0}^{t} e^{\left(b_{1}+\tilde{b}\right) s-\mu \delta\left(\theta_{s-t} \omega\right)-\mu \int_{0}^{s} \delta\left(\theta_{\tau-t} \omega\right) d \tau} d s \\
& =\left\|v_{0}\right\| e^{-b_{1} t-\mu \delta\left(\theta_{-t} \omega\right)+\mu \int_{-t}^{0} \delta\left(\theta_{s} \omega\right) d s} \\
& \quad+\frac{\tilde{b}^{2}}{a} \int_{0}^{t} e^{-\left(b_{1}+\tilde{b}\right)(t-s)-\mu \delta\left(\theta_{s-t} \omega\right)+\mu \int_{s}^{t} \delta\left(\theta_{\tau-t} \omega\right) d \tau} d s \\
& =\left\|v_{0}\right\| e^{-b_{1} t-\mu \delta\left(\theta_{-t} \omega\right)+\mu \int_{-t}^{0} \delta\left(\theta_{s} \omega\right) d s} \\
& \quad+\frac{\tilde{b}^{2}}{a} \int_{-t}^{0} e^{\left(b_{1}+\tilde{b}\right) s-\mu \delta\left(\theta_{s} \omega\right)+\mu \int_{s}^{0} \delta\left(\theta_{\tau} \omega\right) d \tau} d s \\
& \leq\left\|v_{0}\right\| e^{-b_{1} t-\mu \delta\left(\theta_{-t} \omega\right)+\mu \int_{-t}^{0} \delta\left(\theta_{s} \omega\right) d s} \\
& \quad+\frac{\tilde{b}^{2}}{a} \int_{-\infty}^{0} e^{\left(b_{1}+\tilde{b}\right) s-\mu \delta\left(\theta_{s} \omega\right)+\mu \int_{s}^{0} \delta\left(\theta_{\tau} \omega\right) d \tau} d s .
\end{aligned}
$$

Thanks to Proposition 2, we have

$$
\int_{-\infty}^{0} e^{\left(b_{1}+\tilde{b}\right) s-\mu \delta\left(\theta_{s} \omega\right)+\mu \int_{s}^{0} \delta\left(\theta_{\tau} \omega\right) d \tau} d s<\infty .
$$


Then for any $\xi_{0} \in K\left(\theta_{-t} \omega\right)$, we have

$$
\begin{aligned}
\left\|\eta\left(t ; \theta_{-t} \omega, \xi_{0}\right)\right\|= & e^{\delta(\omega)} \| \psi\left(t ; \theta_{-t} \omega, e^{-\delta\left(\theta_{-t} \omega\right)} \xi_{0} \|\right. \\
\leq & e^{\delta(\omega)} e^{-b_{1} t-\mu \delta\left(\theta_{-t} \omega\right)+\mu \int_{-t}^{0} \delta\left(\theta_{s} \omega\right) d s} \cdot d\left(K\left(\theta_{-t} \omega\right)\right) \\
& +e^{\delta(\omega)} \frac{\tilde{b}^{2}}{a} \int_{-\infty}^{0} e^{\left(b_{1}+\tilde{b}\right) s-\mu \delta\left(\theta_{s} \omega\right)+\mu \int_{s}^{0} \delta\left(\theta_{\tau} \omega\right) d \tau} d s .
\end{aligned}
$$

Notice that by Proposition 2

$$
\lim _{t \rightarrow \infty} e^{-b_{1} t-\mu \delta\left(\theta_{-t} \omega\right)+\mu \int_{-t}^{0} \delta\left(\theta_{s} \omega\right) d s}=0 .
$$

For any $\varepsilon>0$ denote by

$$
r_{\varepsilon}(\omega):=e^{\delta(\omega)} \frac{\tilde{b}^{2}}{a} \int_{-\infty}^{0} e^{\left(b_{1}+\tilde{b}\right) s-\mu \delta\left(\theta_{s} \omega\right)+\mu \int_{s}^{0} \delta\left(\theta_{\tau} \omega\right) d \tau} d s+\varepsilon .
$$

Then $\Gamma_{\varepsilon}(\omega)=\overline{\mathbb{B}\left(0, r_{\varepsilon}(\omega)\right)}$ is a closed absorbing set. It remains to show the temperedness of $\Gamma_{\varepsilon}$. In fact, for any $\beta>0$,

$$
\begin{aligned}
e^{\beta t} r\left(\theta_{-t} \omega\right) & =e^{-\beta t}\left(e^{\delta\left(\theta_{-t} \omega\right)} \frac{\tilde{b}^{2}}{a} \int_{-\infty}^{0} e^{\left(b_{1}+\tilde{b}\right) s-\mu \delta\left(\theta_{s-t} \omega\right)+\mu \int_{s}^{0} \delta\left(\theta_{\tau-t} \omega\right) d \tau} d s+\varepsilon\right) \\
& =e^{-\beta t}\left(e^{\delta\left(\theta_{-t} \omega\right)} \frac{\tilde{b}^{2}}{a} \int_{-\infty}^{0} e^{\left(b_{1}+\tilde{b}\right)(s+t)-\mu \delta\left(\theta_{s} \omega\right)+\mu \int_{s}^{-t} \delta\left(\theta_{\tau} \omega\right) d \tau} d s+\varepsilon\right) \\
& \rightarrow 0 \quad \text { as } t \rightarrow \infty
\end{aligned}
$$

The proof is complete.

Lemma 3, Lemma 4, together with Proposition 1 and Remark 1 give immediately the following Theorem.

Theorem 3 The random dynamical system generated by system (6) - (8) possesses a unique global random attractor provided that

$$
m_{1}-m_{2}+a_{21}-a_{12}>0 \quad \text { and } \quad a_{31}-a_{13}>0 .
$$

\section{Closing remarks}

This work is devoted to study the long term behavior of a predator prey model with indirect effects under random environments. The main goal is to make use of the theory of random dynamical systems to study the existence and properties of the pullback random attractor. The concept of "pullback" fits well in this model, as well as other general population models, while we are observing a system at its present state, that has been running for a long time starting from the past. 
In particular, we investigated two different versions of systems evolving in random environments, one concerning random parameters, the other concerning multiplicative white noise. For both systems we were able to prove the existence of a unique global random attractor. Interestingly, the conditions

$$
m_{1}-m_{2}+a_{21}-a_{12}>0 \text { and } a_{31}-a_{13}>0
$$

ensure that both the random system and the stochastic system have a unique global random attractor, although obtained by different techniques. For the random system, we were also able to obtain conditions under which the system is persistent, i.e., the three species coexist.

The concept of random attractors can reflect the interactions between the species with their randomly varying environments. The idea of describing long term dynamics of biological models by using random attractors or more specifically random trajectories can be applied equally well in population genetics, ecology and eco-evolutionary dynamics, as well as any area of applied sciences that involve dynamics over time subject to random environments.

Needless to say that we have chosen two concrete situations to illustrate how the mathematical theory of random dynamical systems is suitable to analyze environmental effects on nonlinear dynamical systems. Several other possibilities can be considered, and in fact we plan to do it in future. For instance, is there any substantial difference in considering the Ito interpretation of the noise instead of the Stratonovich one considered in this paper? We note that the analysis of the Ito version is analogous if the coefficients $b_{j}$ are modified appropriately. This, of course, changes the conditions for the existence of pullback attractor which will depend also on the intensity of the noise, that is the parameter $\mu$. The choice of Ito or Stratonovich noise is essentially a modeling issue rather than a mathematical one.

It is well known that both kind of noise can produce completely different asymptotic behavior, and this is why it is interesting to carry out an analysis of this point. On the other side, one may consider also different noises appearing in each coefficient what will yield to a more involved framework which is worth being analyzed as well. In summary, the analysis performed in the paper is just the beginning of a long time program in which we will put our efforts over the next years.

Acknowledgement. We sincerely thank the referees for the helpful and interesting comments which allowed us to improve the presentation of this paper.

\section{References}

1. L. Arnold, Random Dynamical Systems. Springer-Verlag, Berlin 1998.

2. Y. Asai and P.E. Kloeden, Numerical schemes for random ODEs via stochastic differential equations. Commun. Appl. Analysis, 17 (2013), no. 3 \& 4, 521-528. 
3. B. Bolker, M. Holyoak, V. Krivan, L. Rowe and O. Schmitz, Connecting theoretical and empirical studies of trait-mediated interactions. Ecology, 84 (2003), 1101-1114.

4. J. L. Brooks and I. D. Stanley, Predation, body size, and composition of plankton. Science, 150.3692 (1965) 23-35.

5. T. Caraballo, M.J. Garrido-Atienza, B. Schmalfuß and J. Valero, Non-autonomous and random attractors for delay random semilinear equations without uniqueness. Discrete Contin. Dyn. Syst., 21 (2008), no. 2, 415-443.

6. D. Cariveau, R.E. Irwin, A.K. Brody, S.L. Garcia-Mayeya and A. Von der Ohe, Direct and indirect effects of pollinators and seed predators to selection on plant and floral traits. OIKOS 104 (2004), 15-26.

7. T. Caraballo, R. Colucci, X. Han, Non-autonomous Dynamics of a Semi-Kolmogorov Population Model with Periodic Forcing. (under review).

8. T. Caraballo, K. Lu, Attractors for stochastic lattice dynamical systems with a multiplicative noise, Front. Math. China, 3 (2008), no. 3, 317-335.

9. T. Caraballo, G. Lukaszewicz and J. Real. Pullback attractors for asymptotically compact nonautonomous dynamical systems. Nonlinear Analysis TMA 6 (2006), 484-498.

10. R. Colucci, Coexistence in a One-Predator, Two-Prey system with Indirect Effects. Journal of Applied Mathematics, (2013), Article ID 625391, 13 pages http://dx.doi.org/10.1155/2013/625391

11. R. Colucci, and D. Nunez, Periodic Orbits for a Three-Dimensional Biological Differential Systems. Abstract and Applied Analysis, (2013), Article ID 465183, 10 pages, http://dx.doi.org/10.1155/2013/465183

12. Indirect effects affects ecosystem dynamics. http://www.ictp-saifr.org, 2011.

13. F. Flandoli and B. Schmalfuß. Random attractors for the 3D stochastic Navier-Stokes equation with multiplicative noise. Stochastics Stochastics Rep., 59 (1996), no. 1-2, $21-45$.

14. P. E. Kloeden and M. Rasmussen. Nonautonomous Dynamical Systems, American Mathematical Society, Providence, RI, 2011

15. B. A. Menge, Indirect effects in marine rocky intertidal interaction webs: patterns and importance. Ecological Monographs 65, 1995.

16. K. Rohde, Nonequilibrium Ecology. Cambridge University Press, 2005.

17. M. R. Walsh, D. N. Reznick, Interactions between the direct and indirect effects of predators determine life history evolution in a killifish. Pnas, www.pnas.org/cgi/doi/10.1073/pnas.0710051105.

18. J. T. Wootton, Indirect Effects, Prey Susceptibility, and Habitat Selection: Impacts of Birds on Limpets and Algae. Ecology, 73 (1992), no. 3, 981-991 . 